\title{
Cycles of arbitrary length in distance graphs on $\mathbb{F}_{q}^{d}$
}

\author{
A. Iosevich, G. Jardine and B. McDonald
}

\begin{abstract}
For $E \subset \mathbb{F}_{q}^{d}, d \geq 2$, where $\mathbb{F}_{q}$ is the finite field with $q$ elements, we consider the distance graph $\mathcal{G}_{t}^{\text {dist }}(E), t \neq 0$, where the vertices are the elements of $E$, and two vertices $x$, $y$ are connected by an edge if $\|x-y\| \equiv\left(x_{1}-y_{1}\right)^{2}+\cdots+\left(x_{d}-y_{d}\right)^{2}=t$. We prove that if $|E| \geq C_{k} q^{\frac{d+2}{2}}$, then $\mathcal{G}_{t}^{\text {dist }}(E)$ contains a statistically correct number of cycles of length $k$. We are also going to consider the dot-product graph $\mathcal{G}_{t}^{\text {prod }}(E), t \neq 0$, where the vertices are the elements of $E$, and two vertices $x, y$ are connected by an edge if $x \cdot y \equiv x_{1} y_{1}+\cdots+x_{d} y_{d}=t$. We obtain similar results in this case using more sophisticated methods necessitated by the fact that the function $x \cdot y$ is not translation invariant. The exponent $\frac{d+2}{2}$ is improved for sufficiently long cycles.
\end{abstract}

\section{CONTENTS}

1. Introduction

2. Paths on the Dot Product Graph of $E$

3. Proof of the Main Theorem 9

4. Existence of Non-Degenerate Cycles 18

References 22

\section{Introduction}

Let $\mathbb{F}_{q}$ denote the finite field with $q$ elements, and let $\mathbb{F}_{q}^{d}$ denote the $d$-dimensional vector space over this field. Given $E \subset \mathbb{F}_{q}^{d}, d \geq 2$, define the distance graph $\mathcal{G}_{t}^{\text {dist }}(E), t \neq 0$ by letting the points in $E$ be vertices and connecting two vertices $x$ and $y$ by an edge if

$$
\|x-y\| \equiv \sum_{i=1}^{d}\left(x_{i}-y_{i}\right)^{2}=t .
$$

The research of the first listed author was partially supported by the National Science Foundation grant no. HDR TRIPODS - 1934962. 
Similarly, define the dot product graph $\mathcal{G}_{t}^{\text {prod }}(E), t \neq 0$ by letting the points in $E$ be vertices and connecting two vertices $x$ and $y$ by an edge if

$$
x \cdot y \equiv \sum_{i=1}^{d} x_{i} y_{i}=t .
$$

Considerable progress has been achieved in the study of $\mathcal{G}_{t}^{\text {dist }}(E)$ and $\mathcal{G}_{t}^{\text {prod }}(E)$ over the years. See, for example, [1, [2, [3, 6], 7], 12, 13, 14, 15, 20, 25, and others. In [15, the first listed author and Rudnev proved that if $E \subset \mathbb{F}_{q}^{d}, d \geq 2$, and $t \neq 0$, then

$$
|\{(x, y) \in E \times E:|| x-y||=t\}|=|E|^{2} q^{-1}+D(E),
$$

where

$$
|D(E)| \leq 2 q^{\frac{d-1}{2}}|E| .
$$

In particular, this implies that if $t \neq 0$ and $|E|$ is larger than $4 q^{\frac{d+1}{2}}$, then the number of edges in $\mathcal{G}_{t}^{\text {dist }}(E)$ is at least $\frac{1}{2}|E|^{2} q^{-1}$, and as the size of $|E|$ increases, the number of edges approaches $|E|^{2} q^{-1}$.

In 12], Derrick Hart and the first listed author proved that if $t \neq 0$ and $E \subset \mathbb{F}_{q}^{d}$, then

$$
|\{(x, y) \in E \times E: x \cdot y=t\}|=|E|^{2} q^{-1}+R(E),
$$

where

$$
|R(E)| \leq q^{\frac{d-1}{2}}|E|
$$

Once again, this means that the number of edges in $\mathcal{G}_{t}^{\text {prod }}(E)$ approaches $|E|^{2} q^{-1}$ as $|E|$ increases above $q^{\frac{d+1}{2}}$.

Definition 1.1. Let $C_{n}^{\text {dist }}$ be the number of cycles of length $n$ in $\mathcal{G}_{t}^{\text {dist }}$, i.e.

$$
C_{n}^{\text {dist }}=\left|\left\{\left(x_{1}, x_{2}, \ldots, x_{n}\right) \in E^{n}:\left\|x_{1}-x_{2}\right\|=|| x_{2}-x_{3}\left\|=\cdots=|| x_{n}-x_{1}\right\|\right\}\right|
$$

and similarly let $C_{n}^{\text {prod }}$ be the number of cycles of length $n$ in $\mathcal{G}_{t}^{\text {prod }}$.

Our main results are to estimate $C_{n}^{\text {dist }}, C_{n}^{\text {prod }}$. Heuristically since there are $|E|^{n} n$-tuples of vertices in $\mathcal{G}_{t}^{\text {dist }}$ and $\mathcal{G}_{t}^{\text {prod }}$, and a randomly chosen pair of points in $\mathbb{F}_{q}^{d}$ will have distance, dotproduct, respectively, $t$ with probability $\sim 1 / q$, we expect that $C_{n}^{\text {prod }} \sim q^{-n}|E|^{n}$ when $E$ is large. This is made precise by the following theorem.

THEOREM 1.2. Let

$$
\gamma= \begin{cases}-1 & : d=2 \\ -\frac{d-2}{2} & : d \geq 3\end{cases}
$$

If

$$
12 q^{\gamma}+8 \frac{q^{d+2}}{|E|^{2}}+\left(24+12\left\lfloor\frac{n}{2}\right\rfloor\right) \frac{q^{\frac{d+1}{2}}}{|E|} \leq 1
$$

then for $n \geq 6$,

$$
\left|C_{n}^{d i s t}-\frac{|E|^{n}}{q^{n}}\right| \leq \frac{|E|^{n}}{q^{n}}\left(12 q^{\gamma}+8 \frac{q^{d+1}}{|E|^{2}}+\left(24+12\left\lfloor\frac{n}{2}\right\rfloor\right) \frac{q^{\frac{d+1}{2}}}{|E|}\right)
$$


Moreover,

$$
\begin{aligned}
& \left|C_{4}^{d i s t}-\frac{|E|^{4}}{q^{4}}\right| \leq \frac{|E|^{4}}{q^{4}}\left(12 q^{\gamma}+8 \frac{q^{d+2}}{|E|^{2}}+28 \frac{q^{\frac{d+1}{2}}}{|E|}\right) \\
& \left|C_{5}^{d i s t}-\frac{|E|^{5}}{q^{5}}\right| \leq \frac{|E|^{5}}{q^{5}}\left(12 q^{\gamma}+8 \frac{q^{\frac{2 d+3}{2}}}{|E|^{2}}+32 \frac{q^{\frac{d+1}{2}}}{|E|}\right)
\end{aligned}
$$

The same is true for $C_{n}^{\text {prod }}$.

This theorem says that if $|E|$ is much bigger than $q^{\frac{d+2}{2}}$ for $q$ sufficiently large, then $C_{n}^{\text {dist }}, C_{n}^{\text {prod }}$ are very close to $\frac{|E|^{n}}{q^{n}}$. For the case $n=4$, we cannot get a nontrivial result without the restriction on the size of $E$. However, for larger $n$ we can accept a weaker restriction on the size of $E$, at the cost of a weaker error term. The techniques for proving these theorems are essentially the same.

Theorem 1.3. For $n \geq 5$ and $q$ sufficiently large,

$$
\left|C_{n}^{\text {dist }}-\frac{|E|^{n}}{q^{n}}\right| \leq\left(36+80 \cdot 6^{\left\lfloor\frac{n}{2}\right\rfloor-2}+12\left\lfloor\frac{n}{2}\right\rfloor\right) \frac{|E|^{n}}{q^{n}} q^{-\left(\frac{n}{2}-1\right) \delta}
$$

whenever

$$
|E| \geq \begin{cases}q^{\frac{1}{2}\left(d+2-\frac{k-2}{k-1}+\delta\right)} & : n=2 k, \text { even } \\ q^{\frac{1}{2}\left(d+2-\frac{2 k-3}{2 k-1}+\delta\right)} & : n=2 k+1 \text { odd }\end{cases}
$$

where

$$
0<\delta<\frac{1}{2\left\lfloor\frac{n}{2}\right\rfloor^{2}}
$$

Our final variant of Theorem 1.2 is to count non-degenerate cycles.

Definition 1.4. Let $\mathcal{N}_{n}^{\text {dist }}$ resp. $\mathcal{N}_{n}^{\text {prod }}$ be the number of non-degenerate cycles in $\mathcal{G}_{t}^{\text {dist }}, \mathcal{G}_{t}^{\text {prod }}$, respectively, i.e. cycles $x_{1}, \ldots, x_{n}$ with $x_{i} \neq x_{j}$ when $i \neq j$.

TheOrem 1.5. For $n \geq 4$ and $q$ sufficiently large, if

$$
|E| \geq \begin{cases}q^{\frac{1}{2}\left(d+2-\frac{k-2}{k-1}+\delta\right)} & : n=2 k \\ q^{\frac{1}{2}\left(d+2-\frac{2 k-3}{2 k-1}+\delta\right)} & : n=2 k+1\end{cases}
$$

then

$$
\left|\mathcal{N}_{n}^{\text {dist }}-\frac{|E|^{n}}{q^{n}}\right| \leq \frac{|E|^{n}}{q^{n}}\left(K_{n} q^{-\left(\frac{n}{2}-1\right) \delta}+2 n q^{-\frac{2}{n-1}}+c_{n} q^{-\frac{d-3}{2}-\varepsilon}\right)
$$

where $K_{4}=48$, and

$$
\begin{aligned}
K_{n} & =36+80 \cdot 6^{\left\lfloor\frac{n}{2}\right\rfloor-2}+12\left\lfloor\frac{n}{2}\right\rfloor \text { for } n \geq 5 \\
c_{n} & =(n-1)^{n-3} \cdot 2^{\left(\begin{array}{c}
n-1 \\
2
\end{array}\right)-n+3} \\
\varepsilon & = \begin{cases}1-\frac{k-2}{k-1}+\delta & : n=2 k \\
1-\frac{2 k-3}{2 k-1}+\delta & : n=2 k+1\end{cases}
\end{aligned}
$$

The same estimates hold for $\mathcal{N}_{n}^{\text {prod }}$. 
REMARK 1.6. A close variant of Theorem 1.5 was proved in 14 in the context of the distance graph with a weaker exponent, namely $\frac{d+3}{2}$ in place of $\frac{d+2}{2}$ above. Moreover, we get a better exponent than $\frac{d+2}{2}$ above for long cycles. The main result in [14] handles more general configurations and this raises the question of whether the techniques of this paper can be used obtain results for general configurations. We shall address this issue in the sequel.

The proof of Theorem 1.2 requires an estimate for the number of paths of a given length in $\mathcal{G}_{t}^{\text {dist }}, \mathcal{G}_{t}^{\text {prod }}$. This has been done for distances in [1], and we will cover the dot-product case in section 2. See also [22, where an improvement on the length of the possible paths is obtained. To count non-degenerate cycles we will also need to consider the number of embeddings of an arbitrary tree $T$ into $\mathcal{G}_{t}^{\text {dist }}, \mathcal{G}_{t}^{\text {prod }}$, which is handled in section 4 The following result is sufficient to show existence of non-degenerate cycles for $d \geq 3$ under the hypotheses of Theorem 1.5

Theorem 1.7. Fix a tree $T$ with $r+1$ vertices and hence $r$ edges. For $\epsilon>0$, if $|E|>q^{\frac{d+1}{2}+\varepsilon}$ then there is a subset $E^{\prime} \subseteq E$ with

$$
\left|E \backslash E^{\prime}\right| \leq 2 q^{-\frac{2 \varepsilon}{r+1}}|E|
$$

and if $n_{T}$ is the number of embeddings of $T$ into $\mathcal{G}_{t}^{\text {dist }}\left(E^{\prime}\right)$, then

$$
\left|n_{T}-\frac{|E|^{r+1}}{q^{r}}\right| \leq 8 \frac{|E|^{r+1}}{q^{r}} q^{-\frac{2 \epsilon}{r+1}}
$$

The same is true replacing $\mathcal{G}_{t}^{\text {dist }}$ with $\mathcal{G}_{t}^{\text {prod }}$.

We note that in this paper, we obtain the same results for the distance graph and the dot product graph. While the techniques are, at least superficially, somewhat different due to the lack of translation invariance in the dot product setting, it is reasonable to ask whether a general formalism is possible. The underlying edge operator in the distance graph is

$$
A_{t} f(x)=\sum_{\|x-y\|=t} f(y)
$$

while the edge operator in the dot product case is

$$
R_{t} f(x)=\sum_{x \cdot y=t} f(y) d y
$$

The Euclidean variant of $A f(x)$ is

$$
\mathcal{A}_{t} f(x)=\int f(x-y) d \sigma(y)
$$

where $\sigma$ the surface measure on $S^{d-1}$, the unit sphere. The Euclidean variant of $R f(x)$ is the classical Radon transform

$$
\mathcal{R}_{t} f(x)=\int_{x \cdot y=t} \psi(y) f(y) d \sigma_{x, t}(y),
$$

where $\psi$ is a smooth cut-off function and $\sigma_{x . t}$ is the surface measure on $\left\{y \in \mathbb{R}^{d}: x \cdot y=t\right\}$. If $t \neq 0$, both $\mathcal{A}_{t}$ and $\mathcal{R}_{t}$ map $L^{2}\left(\mathbb{R}^{d}\right)$ to $H^{\frac{d-1}{2}}\left(\mathbb{R}^{d}\right)$, where $H^{s}\left(\mathbb{R}^{d}\right)$ is the Sobolev space of $L^{2}\left(\mathbb{R}^{d}\right)$ function with generalized derivative of order $s>0$ in $L^{2}\left(\mathbb{R}^{d}\right.$. See, for example, $2 \mathbf{2 3}$ and the references contained therein. 
A significant amount of progress has been made in the Euclidean setting in studying general configuration problems from the point of view of Sobolev estimate. See, for example, [9, [10 and 16 for some recent work in this direction. It would be interesting to encode the bounds in the finite field setting using a suitable formalism analogous to their Euclidean counterparts. Both the edge operator $A_{t}$ and $R_{t}$, defined above, satisfy the following bounds that we encode as follows. Let

$$
T_{t}^{\phi} f(x)=\sum_{\phi(x, y)=t} f(y)
$$

where $\phi: \mathbb{F}_{q}^{d} \times \mathbb{F}_{q}^{d} \rightarrow \mathbb{F}_{q}$, a function. See 21] for the description of the continuous analog of this family of operators, introduced by Phong and Stein.

Note that if $\phi(x, y)=\|x-y\|$, we recover the operator $A_{t}$, while if $\phi(\delta, y)=x \cdot y$, we cover $R_{t}$. Let

$$
T_{t, 0}^{\phi} f(x)=T_{t}^{\phi} f(x)-q^{-d} \sum_{x \in \mathbb{F}_{q}^{d}} T_{t}^{\phi} f(x),
$$

which amounts to stripping $T f(x)$ of its 0 'th Fourier coefficient. Both $A_{t}$ and $R_{t}$ above satisfy the bound

$$
<T_{t, 0}^{\phi} f, g>\leq C q^{\frac{d-1}{2}}\|f\|_{L^{2}\left(\mathbb{F}_{q}^{d}\right)} \cdot\|g\|_{L^{2}\left(\mathbb{F}_{q}^{d}\right)}
$$

where

$$
\|f\|_{L^{2}\left(\mathbb{F}_{q}^{d}\right)}^{2}=\sum_{x \in \mathbb{F}_{q}^{d}}|f(x)|^{2},
$$

and the inner product on the left hand side above is the $L^{2}\left(\mathbb{F}_{q}^{d}\right)$ inner product.

The estimate (1.5) can be viewed as an analog of the $L^{2}\left(\mathbb{R}^{d}\right) \rightarrow H^{\frac{d-1}{2}}\left(\mathbb{R}^{d}\right)$ bound in the Euclidean case since for any $\phi$ satisfying

$$
\left|\left\{x \in \mathbb{F}_{q}^{d}: \phi(x, y)=t\right\}\right|=\left|\left\{y \in \mathbb{F}_{q}^{d}: \phi(x, y)=t\right\}\right| \approx q^{d-1},
$$

the estimate (1.5) holds with $q^{\frac{d-1}{2}}$ replaced by $q^{d-1}$. It is reasonable to summarize the above using the following notion.

Definition 1.8. Let $T_{t}^{\phi}, T_{t, 0}^{\phi}$ be as above. Suppose that (1.6) holds and, in place of (1.5) we have

$$
<T_{t, 0}^{\phi} f, g>\leq C q^{d-1} q^{-\alpha}\|f\|_{L^{2}\left(\mathbb{F}_{q}^{d}\right)} \cdot\|g\|_{L^{2}\left(\mathbb{F}_{q}^{d}\right)}
$$

for some $\alpha>0$.

Then we say that $T_{t, 0}^{\phi}$ is smoothing of order $\alpha$.

Given a function $\phi: \mathbb{F}_{q}^{d} \times \mathbb{F}_{q}^{d}$, as above, and $E \subset \mathbb{F}_{q}^{d}$, define $\mathcal{G}_{t}^{\phi}(E)$ to be the graph where the vertices are given by the points of $E$, and two vertices are connected by an edge of $\phi(x, y)=t$. In the sequel, we shall engage in a systematic study of the properties of this graph under the smoothing assumption (1.7) and the size assumption (1.6) above.

1.1. Acknowledgements. This paper is dedicated to Professor Vinogradov's 130th birthday. The authors wish to make it clear that they are celebrating Vinogradov's mathematical legacy. In particular, this submission should not be viewed as an endorsement, in any way, of Vinogradov's political and social views. 


\section{Paths on the Dot Product Graph of $E$}

Definition 2.1. Let $\mathcal{P}_{k}$ be the number of paths of length $k$ on $\mathcal{G}_{t}^{\text {prod }}(E)$.

TheOrem 2.2. If $|E|>\frac{k}{\log 2} q^{\frac{d+1}{2}}$, then

$$
\left|\mathcal{P}_{k}-\frac{|E|^{k+1}}{q^{k}}\right| \leq \frac{k}{\log 2} q^{\frac{d+1}{2}} \frac{|E|^{k}}{q^{k}}
$$

We present an argument similar to the proof of Theorem 1.1 in [1]. First we state Theorem 2.1 from [7]:

TheOREM 2.3. For non-negative functions $f, g$ on $\mathbb{F}_{q}^{d}$,

$$
\sum_{x \cdot y=t} f(x) g(y)=q^{-1}\|f\|_{1}\|g\|_{1}+R(t)
$$

where

$$
|R(t)| \leq\|f\|_{2}\|g\|_{2} q^{\frac{d-1}{2}}
$$

if $t \neq 0$.

Note that when $f, g$ are both the indicator function of $E$, this reduces to the previously mentioned result from [12]. Now we will obtain recursive estimates for $\mathcal{P}_{k}$, which are slightly different in the even and odd cases. The induction works by decomposing a path into two paths of about half the length, joined together by an additional edge.

LEMMA 2.4.

$$
\begin{aligned}
\mathcal{P}_{2 k+1} & =q^{-1} \mathcal{P}_{k}^{2}+R_{2 k+1}, \\
\mathcal{P}_{2 k} & =q^{-1} \mathcal{P}_{k} \mathcal{P}_{k-1}+R_{2 k}
\end{aligned}
$$

where

$$
\begin{aligned}
\left|R_{2 k+1}\right| & \leq q^{\frac{d-1}{2}} \mathcal{P}_{2 k}, \\
\left|R_{2 k}\right| & \leq q^{\frac{d-1}{2}} \sqrt{\mathcal{P}_{2 k} \mathcal{P}_{2 k-2}}
\end{aligned}
$$

Proof. Let

$$
\begin{aligned}
f_{1}(x) & =\sum_{y} E(x) E(y) D_{t}(x, y), \\
f_{k+1}(x) & =\sum_{y} E(x) f_{k}(y) D_{t}(x, y),
\end{aligned}
$$

where $D_{t}(x, y)=1$ when $x \cdot y=t$, and is 0 otherwise. Note that $f_{k}(x)$ is the number of paths of length $k$ in $\mathcal{G}_{t}^{\text {prod }}$ starting at $x$. Then by Theorem 2.3 ,

$$
\begin{aligned}
\mathcal{P}_{2 k+1} & =\sum_{x, y} f_{k}(x) f_{k}(y) D_{t}(x, y)=q^{-1}\left(\sum_{x} f_{k}(x)\right)^{2}+R_{2 k+1} \\
& =q^{-1} \mathcal{P}_{k}^{2}+R_{2 k+1}
\end{aligned}
$$


with

$$
\left|R_{2 k+1}\right| \leq q^{\frac{d-1}{2}}\left(\sum_{x} f_{k}(x)^{2}\right)=q^{\frac{d-1}{2}} \mathcal{P}_{2 k}
$$

Similarly, we have

$$
\begin{aligned}
\mathcal{P}_{2 k} & =\sum_{x, y} f_{k}(x) f_{k-1}(y) D_{t}(x, y)=q^{-1}\left(\sum_{x} f_{k}(x)\right)\left(\sum_{y} f_{k-1}(y)\right)+R_{2 k} \\
& =q^{-1} \mathcal{P}_{k} \mathcal{P}_{k-1}+R_{2 k}
\end{aligned}
$$

with

$$
\left|R_{2 k}\right| \leq q^{\frac{d-1}{2}}|| f_{k}\left\|_{2}|| f_{k-1}\right\|_{2}=q^{\frac{d-1}{2}} \sqrt{\mathcal{P}_{2 k} \mathcal{P}_{2 k-2}}
$$

Since the bound for the error term $R_{k}$ still may depend on $\mathcal{P}_{k}, \mathcal{P}_{k-1}, \mathcal{P}_{k-2}$, we will need an upper bound on $\mathcal{P}_{k}$ for this to be useful.

Lemma 2.5. Let

$$
X=\frac{|E|+q^{\frac{d+1}{2}}}{q}
$$

Then

$$
\mathcal{P}_{k} \leq|E| X^{k}
$$

Proof. We proceed by induction, noting that the case $k=1$ follows from Theorem 2.3 For the odd case, we get

$$
\begin{aligned}
\mathcal{P}_{2 k+1} & \leq q^{-1} \mathcal{P}_{k}^{2}+q^{\frac{d-1}{2}} \mathcal{P}_{2 k} \leq q^{-1}|E|^{2} X^{2 k}+q^{\frac{d-1}{2}}|E| X^{2 k} \\
& =|E| X^{2 k+1}
\end{aligned}
$$

For the even case,

$$
\left|\mathcal{P}_{2 k}-q^{-1} \mathcal{P}_{k} \mathcal{P}_{k-1}\right| \leq q^{\frac{d-1}{2}} \sqrt{\mathcal{P}_{2 k} \mathcal{P}_{2 k-2}}
$$

Squaring and then completing the square to solve for $\mathcal{P}_{2 k}$, we find that

$$
\left(\mathcal{P}_{2 k}-\frac{1}{2} q^{d-1} \mathcal{P}_{2 k-2}-q^{-1} \mathcal{P}_{k} \mathcal{P}_{k-1}\right)^{2} \leq \frac{1}{4} q^{2 d-2} \mathcal{P}_{2 k-2}^{2}+q^{d-2} \mathcal{P}_{k} \mathcal{P}_{k-1} \mathcal{P}_{2 k-2}
$$

And thus

$$
\begin{aligned}
\mathcal{P}_{2 k} & \leq q^{-1} \mathcal{P}_{k} \mathcal{P}_{k-1}+\frac{1}{2} q^{d-1} \mathcal{P}_{2 k-2}+\sqrt{\frac{1}{4} q^{2 d-2} \mathcal{P}_{2 k-2}^{2}+q^{d-2} \mathcal{P}_{k} \mathcal{P}_{k-1} \mathcal{P}_{2 k-2}} \\
& \leq \frac{|E|^{2}}{q} X^{2 k-1}+\frac{1}{2} q^{d-1}|E| X^{2 k-2}+\sqrt{\frac{1}{4} q^{2 d-2}|E|^{2} X^{4 k-4}+q^{d-2}|E|^{3} X^{4 k-3}} \\
& =\frac{|E|^{2}}{q} X^{2 k-1}+\frac{1}{2} q^{d-1}|E| X^{2 k-2}\left(1+\sqrt{1+4 q^{-d}|E| X}\right)
\end{aligned}
$$


Now,

$$
1+4 q^{-d}|E| X=\frac{q^{d+1}+4|E|^{2}+4 q^{\frac{d+1}{2}}|E|}{q^{d+1}}=\frac{\left(q^{\frac{d+1}{2}}+2|E|\right)^{2}}{q^{d+1}}
$$

so

$$
\begin{aligned}
\mathcal{P}_{2 k} & \leq \frac{|E|^{2}}{q} X^{2 k-1}+q^{d-1}|E| X^{2 k-2}\left(1+\frac{|E|}{q^{\frac{d+1}{2}}}\right) \\
& =|E| X^{2 k-2}\left(\frac{|E| X}{q}+q^{d-1}+q^{\frac{d-3}{2}}|E|\right) \\
& =|E| X^{2 k-2}\left(\frac{|E|^{2}+q^{\frac{d+1}{2}}|E|}{q^{2}}+q^{d-1}+q^{\frac{d-3}{2}}|E|\right) \\
& =|E| X^{2 k-2}\left(\frac{|E|^{2}+2 q^{\frac{d+1}{2}}|E|+q^{d+1}}{q^{2}}\right) \\
& =|E| X^{2 k}
\end{aligned}
$$

We now have enough control over the error term $R_{k}$ to prove the Theorem.

Proof of Theorem 2.2, The binomial expansion for $X^{k}$ yields

$$
\begin{aligned}
\mathcal{P}_{k} & \leq|E| X^{k}=\frac{E}{q^{k}} \sum_{i=0}^{k}\left(\begin{array}{c}
k \\
i
\end{array}\right)|E|^{k-i} q^{i \frac{d+1}{2}} \\
& =\frac{|E|^{k+1}}{q^{k}}+\frac{q^{\frac{d+1}{2}}|E|}{q^{k}} \sum_{i=1}^{k}\left(\begin{array}{c}
k \\
i
\end{array}\right)|E|^{k-i} q^{(i-1) \frac{d+1}{2}} \\
& \leq \frac{|E|^{k+1}}{q^{k}}+\frac{q^{\frac{d+1}{2}}|E|^{k}}{q^{k}} \sum_{i=1}^{k}\left(\begin{array}{c}
k \\
i
\end{array}\right)\left(\frac{\log 2}{k}\right)^{i-1} \\
& =\frac{|E|^{k+1}}{q^{k}}+\frac{k}{\log 2} q^{\frac{d+1}{2}} \frac{|E|^{k}}{q^{k}}\left(\left(1+\frac{\log 2}{k}\right)^{k}-1\right) \\
& \leq \frac{|E|^{k+1}}{q^{k}}+\frac{k}{\log 2} q^{\frac{d+1}{2}} \frac{|E|^{k}}{q^{k}}
\end{aligned}
$$


We will show that $\mathcal{P}_{k} \geq \frac{|E|^{k+1}}{q^{k}}-\frac{k}{\log 2} q^{\frac{d-1}{2}}|E|$ by induction, noting that the case $k=1$ follows from Theorem 2.3 Suppose that the result holds for indices less than $2 k+1$. Then

$$
\begin{aligned}
\mathcal{P}_{2 k+1} & \geq \frac{\mathcal{P}_{k}^{2}}{q}-q^{\frac{d-1}{2}} \mathcal{P}_{2 k} \\
& \geq q^{-1}\left(\frac{|E|^{k+1}}{q^{k}}-\frac{k}{\log 2} q^{\frac{d+1}{2}} \frac{|E|^{k}}{q^{k}}\right)^{2}-q^{\frac{d-1}{2}}\left(\frac{|E|^{2 k+1}}{q^{2 k}}+\frac{k}{\log 2} q^{\frac{d+1}{2}} \frac{|E|^{2 k}}{q^{2 k}}\right) \\
& =\frac{|E|^{2 k+2}}{q^{2 k+1}}-q^{\frac{d+1}{2}} \frac{|E|^{2 k+1}}{q^{2 k+1}}\left(\frac{2 k}{\log 2}-q^{\frac{d+1}{2}} \frac{k^{2}}{|E|(\log 2)^{2}}+1+\frac{k}{|E| \log 2} q^{\frac{d+1}{2}}\right) \\
& \geq \frac{|E|^{2 k+2}}{q^{2 k+1}}-q^{\frac{d+1}{2}} \frac{|E|^{2 k+1}}{q^{2 k+1}}\left(\frac{2 k}{\log 2}+1\right) \\
& \geq \frac{|E|^{2 k+2}}{q^{2 k+1}}-\frac{2 k+1}{\log 2} q^{\frac{d+1}{2}} \frac{|E|^{2 k+1}}{q^{2 k+1}}
\end{aligned}
$$

For the even case suppose that the result holds for indices less than $2 k$. Note that we have already established the upper bound for all $k$, and in fact for sufficiently large $q$, we can use the weaker upper bound $\mathcal{P}_{k} \leq \frac{1}{\log 2} \frac{|E|^{k+1}}{q^{k}}$ to obtain

$$
\begin{aligned}
\mathcal{P}_{2 k} & \geq \frac{\mathcal{P}_{k} \mathcal{P}_{k-1}}{q}-q^{\frac{d-1}{2}} \sqrt{\mathcal{P}_{2 k} \mathcal{P}_{2 k-2}} \\
& \geq q^{-1}\left(\frac{|E|^{k+1}}{q^{k}}-\frac{k}{\log 2} q^{\frac{d+1}{2}} \frac{|E|^{k}}{q^{k}}\right)\left(\frac{|E|^{k}}{q^{k-1}}-\frac{k-1}{\log 2} q^{\frac{d+1}{2}} \frac{|E|^{k-1}}{q^{k-1}}\right)-\frac{1}{\log 2} q^{\frac{d-1}{2}} \frac{|E|^{2 k}}{q^{2 k-1}} \\
& =\frac{|E|^{2 k+1}}{q^{2 k}}-q^{\frac{d+1}{2}} \frac{|E|^{2 k}}{q^{2 k}}\left(\frac{2 k-1}{\log 2}-q^{\frac{d+1}{2}} \frac{k(k-1)}{|E|(\log 2)^{2}}+\frac{1}{\log 2}\right) \\
& \geq \frac{|E|^{2 k+1}}{q^{2 k}}-\frac{2 k}{\log 2} q^{\frac{d+1}{2}} \frac{|E|^{2 k}}{q^{2 k}}
\end{aligned}
$$

\section{Proof of the Main Theorem}

We first present the following functional theorems for the distance and dot-product case respectively, which will allow us to prove the main theorem inductively.

Theorem 3.1. For nonnegative functions $f, g: \mathbb{F}_{q}^{d} \times \mathbb{F}_{q}^{d} \rightarrow \mathbb{R}$, let $F(x)=\sum_{y} f(x, y), G(z)=$ $\sum_{w} g(z, w), F^{\prime}(y)=\sum_{x} f(x, y), G^{\prime}(w)=\sum_{z} g(z, w)$. Then for nonzero $t \in \mathbb{F}_{q}$,

$$
\begin{aligned}
& \left|\sum_{\|x-z\|=\|w-y\|=t} f(x, y) g(z, w)-q^{-2}\|f\|_{1}\|g\|_{1}\right| \\
& \quad \leq 3 q^{-\frac{d+2}{2}}\|f\|_{1}\|g\|_{1}+4 q^{d-1}\|f\|_{2}\|g\|_{2}+4 q^{\frac{d-3}{2}}\left(\|F\|_{2}\|G\|_{2}+\left\|F^{\prime}\right\|_{2}\left\|G^{\prime}\right\|_{2}\right)
\end{aligned}
$$


In the case $d=2$, one has

$$
\begin{aligned}
& \left|\sum_{\|x-z||=\| w-y \|=t} f(x, y) g(z, w)-q^{-2}\|f\|_{1}\|g\|_{1}\right| \\
& \quad \leq 3 q^{-3}\|f\|_{1}\|g\|_{1}+4 q\|f\|_{2}\|g\|_{2}+4 q^{-\frac{1}{2}}\left(\|F\|_{2}\|G\|_{2}+\left\|F^{\prime}\right\|_{2}\left\|G^{\prime}\right\|_{2}\right)
\end{aligned}
$$

TheOREM 3.2. With the same notation as in Theorem 3.1,

$$
\left|\sum_{x \cdot z=y \cdot w=t} f(x, y) g(z, w)-q^{-2}\|f\|_{1}\|g\|_{1}\right| \leq 2 q^{d-1}\|f\|_{2}\|g\|_{2}+q^{\frac{d-3}{2}}\left(\|F\|_{2}\|G\|_{2}+\left\|F^{\prime}\right\|_{2}\left\|G^{\prime}\right\|_{2}\right)
$$

Definition 3.3. For $t \in \mathbb{F}_{q}$, let $S_{t}=\left\{x \in \mathbb{F}_{q}^{d}:\|x\|=t\right\}$. We will also identify a set with its indicator function, so that $S_{t}(x)=1$ when $x \in S_{t}$.

We will use a few facts about the discrete sphere $S_{t}$. See for example the appendix of [2] for a treatment of a theorem proved by Minkowski at the age of 17 (see [19]), of which the following lemma is a special case.

LEMMA 3.4. When $t \neq 0$,

$$
\begin{aligned}
& \left|S_{t}\right|=q^{d-1}+\mathcal{E}, \text { with } \\
& |\mathcal{E}| \leq q^{\frac{d}{2}}
\end{aligned}
$$

Moreover, in the case $d=2$,

$$
\left|S_{t}\right|=q \pm 1
$$

Lemma 3.5. If $t \neq 0$ and $m \neq 0$, then

$$
\left|\hat{S}_{t}(m)\right| \leq 2 q^{-\frac{d+1}{2}}
$$

Corollary 3.6.

$$
\begin{gathered}
\left|S_{t}\right| \leq 2 q^{d-1}, \text { and } \\
\left|S_{t}\right|^{2}=q^{2 d-2}+\mathcal{E}^{\prime}, \text { with } \\
\left|\mathcal{E}^{\prime}\right|=\left|2 q^{d-1} \mathcal{E}+\mathcal{E}^{2}\right| \leq 3 q^{\frac{3 d-2}{2}}
\end{gathered}
$$

In the case $d=2$,

$$
\left|\mathcal{E}^{\prime}\right| \leq 3 q
$$

Proof of Theorem 3.1. To estimate the relevant sum, by Fourier inversion on $S_{t}$ and then unpacking the definition of $\hat{f}$ and $\hat{g}$ we find that 


$$
\begin{aligned}
\sum_{\|x-z\|=\|w-y\|=t} f(x, y) g(z, w) & =\sum_{x, y, z, w} f(x, y) g(z, w) S_{t}(x-z) S_{t}(y-w) \\
& =\sum_{x, y, z, w} \sum_{m, \ell} \hat{S}_{t}(m) \hat{S}_{t}(\ell) \chi((x-z) \cdot m) \chi((y-w) \cdot \ell) f(x, y) g(z, w) \\
& =q^{4 d} \sum_{m, \ell} \hat{S}_{t}(m) \hat{S}_{t}(\ell) \overline{\hat{f}(m, \ell)} \hat{g}(m, \ell) \\
& =\left(\frac{\left|S_{t}\right|}{q^{d}}\right)^{2}\|f\|_{1}\|g\|_{1}+R_{A}+R_{B}+R_{C} \\
& =q^{-2}\|f\|_{1}\|g\|_{1}+q^{-2 d} \mathcal{E}^{\prime}\|f\|_{1}\|g\|_{1}+R_{A}+R_{B}+R_{C}
\end{aligned}
$$

Where $R_{A}$ corresponds to the terms where $m, \ell \neq 0, R_{B}$ corresponds to the terms where $m \neq 0$ and $\ell=0$, and $R_{C}$ corresponds to the terms where $m=0$ and $\ell \neq 0$. Now Theorem 3.1 is an immediate corollary of the following lemma:

LEMMA 3.7.

$$
\begin{aligned}
& \left|R_{A}\right| \leq 4 q^{d-1}|| f\left\|_{2}\right\| g \|_{2}, \\
& \left|R_{B}\right| \leq 4 q^{\frac{d-3}{2}}\|F\|_{2}\|G\|_{2}, \\
& \left|R_{C}\right| \leq 4 q^{\frac{d-3}{2}}\left\|F^{\prime}\right\|_{2}\left\|G^{\prime}\right\|_{2} .
\end{aligned}
$$

Proof. For $R_{A}$, applying the bound $\left|\hat{S}_{t}(m)\right| \leq 2 q^{-\frac{d+1}{2}}$ for $m \neq 0$ and Cauchy-Schwarz, we get

$$
\begin{aligned}
\left|R_{A}\right| & =\left|q^{4 d} \sum_{m, \ell \neq 0} \hat{S}_{t}(m) \hat{S}_{t}(\ell) \overline{\hat{f}(m, \ell)} \hat{g}(m, \ell)\right| \\
& \leq 4 q^{3 d-1} \sum_{m, \ell \neq 0}|\hat{f}(m, \ell)| \cdot|\hat{g}(m, \ell)| \\
& \leq 4 q^{3 d-1}\|\hat{f}\|_{2} \cdot\|\hat{g}\|_{2}=4 q^{d-1}\|f\|_{2} \cdot\|g\|_{2}
\end{aligned}
$$

For $R_{B}$, observe that

$$
\begin{aligned}
\hat{f}(m, 0) & =q^{-2 d} \sum_{x, y \in \mathbb{F}_{q}^{d}} \chi(-x \cdot m) f(x, y) \\
& =q^{-2 d} \sum_{x} \chi(-m \cdot x) F(x)=q^{-d} \hat{F}(m)
\end{aligned}
$$


and therefore

$$
\begin{aligned}
\left|R_{B}\right| & =\left|q^{4 d} \sum_{m \neq 0} \hat{S}_{t}(m) \hat{S}_{t}(0) \overline{\hat{f}(m, 0)} g(m, 0)\right| \\
& \leq q^{3 d}\left|S_{t}\right| \cdot \max _{m}\left|\hat{S}_{t}(m)\right| \sum_{m \neq 0}|\hat{f}(m, 0)| \cdot|\hat{g}(m, 0)| \\
& =q^{d}\left|S_{t}\right| \cdot \max _{m}\left|\hat{S}_{t}(m)\right| \sum_{m \neq 0}|\hat{F}(m)| \cdot|\hat{G}(m)| \\
& \leq 4 q^{\frac{3 d-3}{2}}|| \hat{F}\left\|_{2} \cdot\right\| \hat{G}\left\|_{2}=4 q^{\frac{d-3}{2}}\right\| F\left\|_{2} \cdot\right\| G \|_{2}
\end{aligned}
$$

The bound for $R_{C}$ follows with the same argument by symmetry.

Proof of Theorem 3.2 ,

$$
\begin{aligned}
\sum_{x \cdot z=y \cdot w=t} f(x, y) g(z, w) & =q^{-2} \sum_{\substack{x, y, z, w \\
\alpha, \beta \in \mathbb{F}_{q}\\
}} f(x, y) g(z, w) \chi(\alpha(x \cdot z-t)) \chi(\beta(y \cdot w-t)) \\
& =q^{-2}\|f\|_{1}\|g\|_{1}+R_{D}+R_{E}+R_{F}
\end{aligned}
$$

Where $R_{D}$ corresponds to the $\alpha, \beta \neq 0$ terms, $R_{E}$ corresponds to $\alpha \neq 0, \beta=0$, and $R_{F}$ corresponds to $\alpha=0, \beta \neq 0$. By Cauchy-Schwarz,

$$
\begin{aligned}
R_{D}^{2} & =\left(q^{-2} \sum_{\substack{x, y, z, w \\
\alpha, \beta \neq 0}} f(x, y) g(z, w) \chi(\alpha(x \cdot z-t)) \chi(\beta(y \cdot w-t))\right)^{2} \\
& \leq q^{-4}\left(\sum_{x, y} f(x, y)^{2}\right) \cdot \mathcal{S}
\end{aligned}
$$

where

$$
\begin{aligned}
\mathcal{S} & =\sum_{\substack{x, y, z, z^{\prime}, w, w^{\prime} \\
\alpha, \alpha^{\prime}, \beta, \beta^{\prime} \neq 0}} \chi(\alpha(x \cdot z-t)) \chi\left(-\alpha^{\prime}\left(x \cdot z^{\prime}-t\right)\right) \chi(\beta(y \cdot w-t)) \chi\left(-\beta^{\prime}\left(y^{\prime} \cdot w^{\prime}-t\right)\right) g(z, w) g\left(z^{\prime}, w^{\prime}\right) \\
& =\sum_{\substack{x, y, z, z^{\prime}, w, w^{\prime} \\
\alpha, \alpha^{\prime}, \beta, \beta^{\prime}}} g(z, w) g\left(z^{\prime}, w^{\prime}\right) \chi\left(x \cdot\left(\alpha z-\alpha^{\prime} z^{\prime}\right)\right) \chi\left(y \cdot\left(\beta w-\beta^{\prime} w^{\prime}\right)\right) \chi\left(t\left(\alpha^{\prime}-\alpha\right)\right) \chi\left(t\left(\beta^{\prime}-\beta\right)\right) \\
& =q^{2 d}\left(\begin{array}{c}
\sum_{\substack{z, z^{\prime}, w, w^{\prime} \\
\alpha, \alpha^{\prime}, \beta, \beta^{\prime} \neq 0 \\
\alpha z=\alpha^{\prime} z^{\prime}, \beta w=\beta^{\prime} w^{\prime}}} g(z, w) g\left(z^{\prime}, w^{\prime}\right) \chi\left(t\left(\alpha^{\prime}-\alpha\right)\right) \chi\left(t\left(\beta^{\prime}-\beta\right)\right) \\
\end{array}\right. \\
& =q^{2 d}(I+I I+I I I+I V) \\
& \lesssim q^{2 d}(I+I I+I I I),
\end{aligned}
$$

where $I$ corresponds to the terms in the sum where $\alpha=\alpha^{\prime}, \beta=\beta^{\prime}, I I$ corresponds to $\alpha \neq \alpha^{\prime}$, $\beta \neq \beta^{\prime}$, III corresponds to $\alpha \neq \alpha^{\prime}, \beta=\beta$, and $I V$ corresponds to $\alpha=\alpha^{\prime}, \beta \neq \beta^{\prime}$. By symmetry, 
$I I I=I V$.

$$
I=\sum_{\substack{\alpha, \beta \neq 0 \\ z, w}} g(z, w)^{2}=q^{2} \sum_{z, w} g(z, w)^{2}
$$

For $I I$ we make the substitution $a=\alpha / \alpha^{\prime},=\alpha^{\prime}, u=\beta / \beta^{\prime}, v=\beta^{\prime}$, and applying Cauchy-Schwarz yields

$$
\begin{aligned}
I I & =\sum_{\substack{z, w \\
a, u \neq 0,1}} \sum_{b, v \neq 0} g(z, w) g(a z, u w) \chi(t b(1-a)) \chi(t v(1-u)) \\
& =\sum_{\substack{z, w \\
a, u \neq 0,1}} g(z, w) g(a z, u w) \leq \sum_{a, u \neq 0,1}\left(\sum_{z, w} g(z, w)^{2}\right)^{\frac{1}{2}}\left(\sum_{z, w} g(a z, u w)^{2}\right)^{\frac{1}{2}} \\
& =\sum_{a, u \neq 0,1} \sum_{z, w} g(z, w)^{2} \leq q^{2} \sum_{z, w} g(z, w)^{2} .
\end{aligned}
$$

We use the same subsitution for $I I I$, noting that $\beta=\beta^{\prime} \neq 0$ forces $u=1$ and $v=\beta$.

$$
\begin{aligned}
|I I I| & =\left|\sum_{\substack{z, w \\
a \neq 0,1 \\
b \neq 0}} \sum_{\beta \neq 0} g(z, w) g(a z, w) \chi(t b(1-a))\right| \\
& =q\left|\sum_{\substack{z, w \\
a \neq 0,1 \\
b \neq 0}} g(z, w) g(a z, w) \chi(t b(1-a))\right| \\
& =q \sum_{\substack{z, w \\
a \neq 0,1}} g(z, w) g(a z, w) \\
& \leq q^{2} \sum_{z, w} g(z, w)^{2}
\end{aligned}
$$

Putting this all together, we have

$$
R_{D} \leq 2 q^{d-1}\|f\|_{2}\|g\|_{2}
$$

Now to estimate $R_{E}$, which corresponds to the terms $\alpha \neq 0, \beta=0$,

$$
\begin{aligned}
R_{E}+q^{-2}\|f\|_{1}\|g\|_{1} & =q^{-2} \sum_{x, y, z, w} \sum_{\alpha \in \mathbb{F}_{q}} \chi(\alpha(x \cdot z-t)) f(x, y) g(z, w) \\
& =q^{-1} \sum_{x, y, z, w} f(x, y) g(z, w) D_{t}(x, z) \\
& =q^{-1} \sum_{x, z} F(x) G(y) D_{t}(x, z)
\end{aligned}
$$


So by Theorem 2.3

$$
\left|R_{E}\right| \leq q^{\frac{d-3}{2}}\|F\|_{2}\|G\|_{2}
$$

By symmetry, the same argument shows that

$$
\left|R_{F}\right| \leq q^{\frac{d-3}{2}}\left\|F^{\prime}\right\|_{2}\left\|G^{\prime}\right\|_{2}
$$

With Theorem 3.1 and Theorem 3.2 the proof of Theorem 1.2 is now a straightforward calculation. In fact, for the rest of the argument the distance and dot-product cases will be unified. For brevity we will use the following notation.

Definition 3.8. Let $P_{k}^{\text {dist }}(x, y)$ resp. $P_{k}^{\text {prod }}(x, y)$ be the number of paths of length $k$ from $x \in E$ to $y \in E$ in $\mathcal{G}_{t}^{\text {dist }}$ resp. $\mathcal{G}_{t}^{\text {prod }}$. We will use $P_{k}^{*}(x, y)$ and $\mathcal{G}_{t}^{*}$ to refer simultaneously to the distance and dot-product versions; $P_{k}^{*}(x, y)$ may essentially be read as $P_{k}^{\text {dist }}(x, y)$ resp. $P_{k}^{\text {prod }}(x, y)$.

Definition 3.9. Furthermore, let $\mathcal{P}_{k}^{*}=\sum_{x, y} P_{k}^{*}(x, y)$, the number of paths of length $k$ from $x$ to $y$ on $\mathcal{G}_{t}^{*}$. Similarly let $C_{k}^{*}$ be the number of cycles of length $k$ on $\mathcal{G}_{t}^{*}$.

DeFinition 3.10. Let

$$
\begin{gathered}
\mathcal{T}_{t}^{\text {dist }}(f, g)=\sum_{\|x-z\|=\|y-w\|=t} f(x, y) g(z, w), \\
\mathcal{T}_{t}^{\text {prod }}(f, g)=\sum_{x \cdot z=y \cdot w=t} f(x, y) g(z, w)
\end{gathered}
$$

and similarly to before $\mathcal{T}_{t}^{*}$ will refer to $\mathcal{T}_{t}^{\text {dist }}$ resp. $\mathcal{T}_{t}^{\text {prod }}$.

Now Theorem 3.1 and Theorem 3.2 can be stated together concisely:

Corollary 3.11 .

$\left|\mathcal{T}_{t}^{*}(f, g)-\frac{1}{q^{2}}\|f\|_{1}\|g\|_{1}\right| \leq 3 q^{-\frac{d+2}{2}}\|f\|_{1}\|g\|_{1}+4 q^{d-1}\|f\|_{2}\|g\|_{2}+4 q^{\frac{d-3}{2}}\left(\|F\|_{2}\|G\|_{2}+\left\|F^{\prime}\right\|_{2}\left\|G^{\prime}\right\|_{2}\right)$ and in the case $d=2$,

$$
\left|\mathcal{T}_{t}^{*}(f, g)-\frac{1}{q^{2}}\|f\|_{1}\|g\|_{1}\right| \leq 3 q^{-3}\|f\|_{1}\|g\|_{1}+4 q^{d-1}\|f\|_{2}\|g\|_{2}+4 q^{\frac{d-3}{2}}\left(\|F\|_{2}\|G\|_{2}+\left\|F^{\prime}\right\|_{2}\left\|G^{\prime}\right\|_{2}\right)
$$

In order to prove Theorem 1.2, we will consider the case when $f(x, y)=P_{k}^{*}(x, y), g(z, w)=$ $P_{\ell}^{*}(z, w)$ for some $k, \ell$.

LEMMA 3.12. Let $f(x, y)=P_{k}^{*}(x, y)$ with $k \geq 2$. If $|E| \geq \frac{2 k}{\log 2} q^{\frac{d+1}{2}}$, then

$$
\begin{aligned}
& \|f\|_{1}=\mathcal{P}_{k}^{*}=\frac{|E|^{k+1}}{q^{k}}+\mathcal{E}_{k} \\
& \|f\|_{2}=\sqrt{C_{2 k}^{*}} \\
& \|F\|_{2}=\left\|F^{\prime}\right\|_{2}=\sqrt{\mathcal{P}_{2 k}^{*}} \leq 2 \frac{|E|^{k+\frac{1}{2}}}{q^{k}}
\end{aligned}
$$

Where

$$
\left|\mathcal{E}_{k}\right| \leq \frac{2 k}{\log 2} q^{\frac{d+1}{2}} \frac{|E|^{k}}{q^{k}}
$$


Proof. Unravel definitions, apply Theorem 2.2 in the dot-product case and the analogous result from [1] in the distance case, and a degree 1 Taylor approximation of $\sqrt{x}$.

Lemma 3.13. Let $\ell \geq k$. If $|E| \geq \frac{2 \ell}{\log 2} q^{\frac{d+1}{2}}$, then

$$
\left|\frac{\left\|P_{k}^{*}\right\|_{1} \cdot\left\|P_{\ell}^{*}\right\|_{1}}{q^{2}}-\frac{|E|^{k+\ell+2}}{q^{k+\ell+2}}\right| \leq(8 k+4 \ell) q^{\frac{d+1}{2}} \frac{|E|^{k+\ell+1}}{q^{k+\ell+2}}
$$

Proof. It follows directly from Theorem 2.2 in the dot-product case and the analogous result from [1] in the distance case that

$$
\left|\frac{\left\|P_{k}^{*}\right\|_{1} \cdot\left\|P_{\ell}^{*}\right\|_{1}}{q^{2}}-\frac{|E|^{k+\ell+2}}{q^{k+\ell+2}}\right| \leq 4(k+\ell) q^{\frac{d+1}{2}} \frac{|E|^{k+\ell+1}}{q^{k+\ell+2}}+16 k \ell q^{d-1} \frac{|E|^{k+\ell}}{q^{k+\ell}}
$$

Using the fact that $q^{\frac{d+1}{2}} \leq \frac{1}{4 \ell}|E|$ finishes the proof of the lemma.

We are now ready to prove the main theorem.

Proof of Theorem 1.2. We proceed by induction. Using Corollary 3.11 and Lemma 3.12 and Lemma 3.13, as well as the estimates from [12] and [15. for the number of edges in $\mathcal{G}_{t}^{*}$, we start by computing $C_{4}^{*}$ and $C_{5}^{*}$. In order to handle the $d=2$ case and the $d \geq 3$ case at once, let

$$
\gamma= \begin{cases}-1 & : d=2 \\ -\frac{d-2}{2} & : d \geq 3\end{cases}
$$

Then we get

$$
\begin{aligned}
\left|C_{4}^{*}-\frac{|E|^{4}}{q^{4}}\right| & =\left|\mathcal{T}_{t}^{*}\left(P_{1}^{*}, P_{1}^{*}\right)-\frac{|E|^{4}}{q^{4}}\right| \leq\left|\mathcal{T}_{t}^{*}\left(P_{1}^{*}, P_{1}^{*}\right)-\frac{|| P_{1}^{*} \|_{1}^{2}}{q^{2}}\right|+\left|\frac{\left.|| P_{1}^{*}\right|_{1} ^{2}}{q^{2}}-\frac{|E|^{4}}{q^{4}}\right| \\
& \leq 3 q^{\gamma-2} \mathcal{P}_{1}^{* 2}+4 q^{d-1} \mathcal{P}_{1}^{*}+8 q^{\frac{d-3}{2}} \mathcal{P}_{2}^{*}+12 q^{\frac{d-7}{2}}|E|^{3} \\
& \leq \frac{|E|^{4}}{q^{4}}\left(12 q^{\gamma}+8 \frac{q^{d+2}}{|E|^{2}}+28 \frac{q^{\frac{d+1}{2}}}{|E|}\right)
\end{aligned}
$$

In particular, under the hypotheses of the theorem we have $C_{4}^{*} \leq 2 \frac{|E|^{4}}{q^{4}}$. Therefore,

$$
\begin{aligned}
\left|C_{5}^{*}-\frac{|E|^{5}}{q^{5}}\right| & =\left|\mathcal{T}_{t}^{*}\left(P_{1}^{*}, P_{2}^{*}\right)-\frac{|E|^{5}}{q^{5}}\right| \leq\left|\mathcal{T}_{t}^{*}\left(P_{1}^{*}, P_{2}^{*}\right)-\frac{\left\|P_{1}^{*}\right\|_{1}|| P_{2}^{*} \|_{1}}{q^{2}}\right|+\left|\frac{\left\|P_{1}^{*}\right\|_{1}\left\|P_{2}^{*}\right\|_{1}}{q^{2}}-\frac{|E|^{5}}{q^{5}}\right| \\
& \leq 3 q^{\gamma-2} \mathcal{P}_{1}^{*} \mathcal{P}_{2}^{*}+4 q^{d-1} \sqrt{\mathcal{P}_{1}^{*} C_{4}^{*}}+8 q^{\frac{d-3}{2}} \sqrt{\mathcal{P}_{2}^{*} \mathcal{P}_{4}^{*}}+16 q^{\frac{d-9}{2}}|E|^{4} \\
& \leq \frac{|E|^{5}}{q^{5}}\left(12 q^{\gamma}+8 \frac{q^{\frac{2 d+3}{2}}}{|E|^{2}}+32 \frac{q^{\frac{d+1}{2}}}{|E|}\right)
\end{aligned}
$$


With an estimate for $C_{4}^{*}$, we can handle any even length inductively:

$$
\begin{aligned}
\left|C_{2 k+2}^{*}-\frac{|E|^{2 k+2}}{q^{2 k+2}}\right| & =\left|\mathcal{T}_{t}^{*}\left(P_{k}^{*}, P_{k}^{*}\right)-\frac{|E|^{2 k+2}}{q^{2 k+2}}\right| \\
& \leq\left|\mathcal{T}_{t}^{*}\left(P_{k}^{*}, P_{k}^{*}\right)-\frac{\left\|P_{k}^{*}\right\|_{1}|| P_{k}^{*} \mid \|_{1}}{q^{2}}\right|+\left|\frac{\left\|P _ { k } ^ { * } \left|\left\|_{1}|| P_{k}^{*}\right\|_{1}\right.\right.}{q^{2}}-\frac{|E|^{2 k+2}}{q^{2 k+2}}\right| \\
& \leq 3 q^{\gamma-2} \mathcal{P}_{k}^{* 2}+4 q^{d-1} C_{2 k}^{*}+8 q^{\frac{d-3}{2}} \mathcal{P}_{2 k}^{*}+12 k q^{\frac{d+1}{2}} \frac{|E|^{2 k+1}}{q^{2 k+2}} \\
& \leq \frac{|E|^{2 k+2}}{q^{2 k+2}}\left(12 q^{\gamma}+8 \frac{q^{d+1}}{|E|^{2}}+(16+12 k) \frac{q^{\frac{d+1}{2}}}{|E|}\right)
\end{aligned}
$$

Finally, noting that under the hypotheses of the theorem we can conclude that $C_{n}^{*} \leq 2 \frac{|E|^{n}}{q^{n}}$ for any even $n \geq 4$, we can handle the odd case:

$$
\begin{aligned}
\left|C_{2 k+1}^{*}-\frac{|E|^{2 k+1}}{q^{2 k+1}}\right| & =\left|\mathcal{T}_{t}^{*}\left(P_{k}^{*}, P_{k-1}^{*}\right)-\frac{|E|^{2 k+1}}{q^{2 k+1}}\right| \\
& \leq\left|\mathcal{T}_{t}^{*}\left(P_{k}^{*}, P_{k-1}^{*}\right)-\frac{|| P_{k}^{*}||_{1}|| P_{k-1}^{*}||_{1}}{q^{2}}\right|+\left|\frac{\left\|P_{k}^{*}\right\|_{1}|| P_{k-1}^{*} \|_{1}}{q^{2}}-\frac{|E|^{2 k+1}}{q^{2 k+1}}\right| \\
& \leq 3 q^{\gamma-2} \mathcal{P}_{k}^{*} \mathcal{P}_{k-1}^{*}+4 q^{d-1} \sqrt{C_{2 k}^{*} C_{2 k-2}^{*}}+8 q^{\frac{d-3}{2}} \sqrt{\mathcal{P}_{2 k}^{*} \mathcal{P}_{2 k-2}^{*}}+(12 k-8) q^{\frac{d+1}{2}} \frac{|E|^{2 k+1}}{q^{2 k+2}} \\
& \leq \frac{|E|^{2 k+1}}{q^{2 k+1}}\left(12 q^{\gamma}+8 \frac{q^{d+1}}{|E|^{2}}+(24+12 k) \frac{q^{\frac{d+1}{2}}}{|E|}\right)
\end{aligned}
$$

The proof for Theorem 1.3 is quite similar, but we will need the following lemma.

Lemma 3.14. Let $\psi_{k}(\alpha)=(k-1) \alpha-k+2$. Then whenever $\frac{k-2}{k-1} \leq \alpha<1$ and $|E| \geq q^{\frac{d+2-\alpha}{2}}$, for sufficiently large $q$ we have

$$
C_{2 k}^{*} \leq A_{k} \frac{|E|^{2 k}}{q^{2 k}} q^{\psi_{k}(\alpha)}
$$

Where $A_{k}=10 \cdot 6^{k-2}$

Proof. For $k=2$, if $|E| \geq q^{\frac{d+2-\alpha}{2}}$ then for sufficiently large $q$,

$$
\begin{aligned}
C_{4}^{*} & \leq \frac{|E|^{4}}{q^{4}}\left(1+12 q^{\gamma}+8 \frac{q^{d+2}}{|E|^{2}}+28 \frac{q^{\frac{d+1}{2}}}{|E|}\right) \\
& \leq \frac{|E|^{4}}{q^{4}}\left(1+12 q^{\gamma}+8 q^{\alpha}+28 q^{\frac{\alpha}{2}-\frac{1}{2}}\right) \leq 10 \frac{|E|^{4}}{q^{4}} q^{\alpha},
\end{aligned}
$$


and so the result holds for $A_{2}=10$. Assuming it holds for $C_{2 k}^{*}$, we find for sufficiently large $q$ that

$$
\begin{aligned}
C_{2 k+2}^{*} & \leq \frac{|E|^{2 k+2}}{q^{2 k+2}}+3 q^{\gamma-2} \mathcal{P}_{k}^{* 2}+4 q^{d-1} C_{2 k}^{*}+8 q^{\frac{d-3}{2}} \mathcal{P}_{2 k}^{*}+12 k q^{\frac{d+1}{2}} \frac{|E|^{2 k+1}}{q^{2 k+2}} \\
& \leq \frac{|E|^{2 k+2}}{q^{2 k+2}}\left(1+12 q^{\gamma}+4 A_{k} \frac{q^{d+1+\psi_{k}(\alpha)}}{|E|^{2}}+(16+12 k) \frac{q^{\frac{d+1}{2}}}{|E|}\right) \\
& \leq \frac{|E|^{2 k+2}}{q^{2 k+2}}\left(1+12 q^{\gamma}+4 A_{k} q^{\psi_{k}(\alpha)+\alpha-1}+(16+12 k) q^{\frac{\alpha}{2}-\frac{1}{2}}\right) \\
& \leq 6 A_{k} \frac{|E|^{2 k+2}}{q^{2 k+2}} q^{\psi_{k+1}(\alpha)}
\end{aligned}
$$

and thus it holds for $C_{2 k+2}$ as long as $\psi_{k+1}(\alpha) \geq 0$, which is the case when $\alpha \geq \frac{k-1}{k}$.

Proof of Theorem 1.3. The argument is similar to the proof of Theorem 1.2. From the estimate for $C_{4}^{*}$, we see that if $|E| \geq q^{\frac{d+2-\alpha}{2}}$ for $0 \leq \alpha<1$, then $C_{4}^{*} \leq A_{2} \frac{|E|^{4}}{q^{4}} q^{\alpha}$. In that case,

$$
\begin{aligned}
\left|C_{5}^{*}-\frac{|E|^{5}}{q^{5}}\right| & \leq 3 q^{\gamma-2} \mathcal{P}_{1}^{*} \mathcal{P}_{2}^{*}+4 q^{d-1} \sqrt{\mathcal{P}_{1}^{*} C_{4}^{*}}+8 q^{\frac{d-3}{2}} \sqrt{\mathcal{P}_{2}^{*} \mathcal{P}_{4}^{*}}+16 q^{\frac{d-9}{2}}|E|^{4} \\
& \leq \frac{|E|^{5}}{q^{5}}\left(12 q^{\gamma}+8 A_{k} \frac{q^{\frac{2 d+3+\alpha}{2}}}{|E|^{2}}+32 \frac{q^{\frac{d+1}{2}}}{|E|}\right)
\end{aligned}
$$

In particular, if $\alpha=\frac{1}{3}-\delta$ for $0<\delta<\frac{2}{9}$, then

$$
\begin{aligned}
\left|C_{5}^{*}-\frac{|E|^{5}}{q^{5}}\right| & \leq \frac{|E|^{5}}{q^{5}}\left(12 q^{\gamma}+8 A_{k} q^{-\frac{3}{2} \delta}+32 q^{-\frac{\delta}{2}-\frac{1}{3}}\right) \\
& \leq\left(44+8 A_{k}\right) \frac{|E|^{5}}{q^{5}} q^{-\frac{3}{2} \delta}
\end{aligned}
$$

For the even case, we use Lemma 3.14 to find that

$$
\begin{aligned}
\left|C_{2 k+2}^{*}-\frac{|E|^{2 k+2}}{q^{2 k+2}}\right| & \leq 3 q^{\gamma-2} \mathcal{P}_{k}^{* 2}+4 q^{d-1} C_{2 k}^{*}+8 q^{\frac{d-3}{2}} \mathcal{P}_{2 k}^{*}+12 k q^{\frac{d+1}{2}} \frac{|E|^{2 k+1}}{q^{2 k+2}} \\
& \leq \frac{|E|^{2 k+2}}{q^{2 k+2}}\left(12 q^{\gamma}+8 A_{k} \frac{q^{d+1+\psi_{k}(\alpha)}}{|E|^{2}}+(16+12 k) \frac{q^{\frac{d+1}{2}}}{|E|}\right)
\end{aligned}
$$

and in particular, if $\alpha=\frac{k-1}{k}-\delta$ for $0<\delta<\frac{1}{2 k^{2}}$ then

$$
\begin{aligned}
\left|C_{2 k+2}^{*}-\frac{|E|^{2 k+2}}{q^{2 k+2}}\right| & \leq \frac{|E|^{2 k+2}}{q^{2 k+2}}\left(12 q^{\gamma}+8 A_{k} q^{-k \delta}+(16+12 k) q^{-\frac{1}{2 k}-\frac{\delta}{2}}\right) \\
& \leq\left(28+8 A_{k}+12 k\right) \frac{|E|^{2 k+2}}{q^{2 k+2}} q^{-k \delta}
\end{aligned}
$$

And finally the odd case; if $\alpha \geq \frac{k-2}{k-1}$, then

$$
\begin{aligned}
\left|C_{2 k+1}^{*}-\frac{|E|^{2 k+1}}{q^{2 k+1}}\right| & \leq 3 q^{\gamma-2} \mathcal{P}_{k}^{*} \mathcal{P}_{k-1}^{*}+4 q^{d-1} \sqrt{C_{2 k}^{*} C_{2 k-2}^{*}}+8 q^{\frac{d-3}{2}} \sqrt{\mathcal{P}_{2 k}^{*} \mathcal{P}_{2 k-2}^{*}}+(12 k-8) q^{\frac{d+1}{2}} \frac{|E|^{2 k+1}}{q^{2 k+2}} \\
& \leq \frac{|E|^{2 k+1}}{q^{2 k+1}}\left(12 q^{\gamma}+8 A_{k} \frac{q^{d+1+\frac{1}{2}((2 k-3) \alpha-2 k+5)}}{|E|^{2}}+(24+12 k) \frac{q^{\frac{d+1}{2}}}{|E|}\right)
\end{aligned}
$$


Note that $\frac{2 k-3}{2 k-1} \geq \frac{k-2}{k-1}$ for all $k$. Thus, if $\alpha=\frac{2 k-3}{2 k-1}-\delta$ for

$$
0<\delta \leq \frac{1}{(k-1)(2 k-1)}
$$

then

$$
\begin{aligned}
\left|C_{2 k+1}^{*}-\frac{|E|^{2 k+1}}{q^{2 k+1}}\right| & \leq \frac{|E|^{2 k+1}}{q^{2 k+1}}\left(12 q^{\gamma}+8 A_{k} q^{-\left(k-\frac{1}{2}\right) \delta}+(24+12 k) q^{-\frac{2}{2 k-1}-\frac{\delta}{2}}\right) \\
& \leq\left(36+8 A_{k}+12 k\right) \frac{|E|^{2 k+1}}{q^{2 k+1}} q^{-\left(k-\frac{1}{2}\right) \delta}
\end{aligned}
$$

\section{Existence of Non-Degenerate Cycles}

The following bound follows directly from Theorem 1.2 in the case $n=4$, and Theorem 1.3 for $n \geq 5$ :

COROllary 4.1. For $n \geq 4$ and $q$ sufficiently large, if

$$
|E| \geq \begin{cases}q^{\frac{1}{2}\left(d+2-\frac{k-2}{k-1}+\delta\right)} & : n=2 k \\ q^{\frac{1}{2}\left(d+2-\frac{2 k-3}{2 k-1}+\delta\right)} & : n=2 k+1\end{cases}
$$

then

$$
\left|C_{n}^{*}-\frac{|E|^{n}}{q^{n}}\right| \leq K_{n} \frac{|E|^{n}}{q^{n}} q^{-\left(\frac{n}{2}-1\right) \delta}
$$

where $K_{4}=48$, and for $n \geq 5 K_{n}=36+80 \cdot 6^{\left\lfloor\frac{n}{2}\right\rfloor-2}+12\left\lfloor\frac{n}{2}\right\rfloor$.

We will show that $\mathcal{G}_{t}^{*}$ admits $\sim \frac{|E|^{k}}{q^{k}}$ non-degenerate $k$-cycles under the hypotheses of Corollary 4.1. By non-degenerate, we mean a cycle in $\mathcal{G}_{t}^{*}$ with $k$ distinct vertices and edges. Our estimates for $C_{k}^{*}$ do not on their own imply the existence of even one non-degenerate cycle, because our estimates include all the degenerate cycles as well as non-degenerate. In order to prove Theorem 1.5. we reduce to a subset of $E$ which we can show admits $\sim \frac{|E|^{k}}{q^{k}}$ non-degenerate $k$-cycles, which implies that $E$ also admits $\sim \frac{|E|^{k}}{q^{k}}$ non-degenerate $k$-cycles.

Definition 4.2. For $\lambda=\lambda(q)>0$ to be specified later (with the property that $\lambda(q) \rightarrow \infty$ ), let

$$
\begin{aligned}
E^{\text {dist }} & =\left\{x \in E: E * S_{t}(x) \leq \lambda \frac{|E|}{q}\right\} \\
E^{\text {prod }} & =\left\{x \in E: \sum_{y} E(y) D_{t}(x, y) \leq \lambda \frac{|E|}{q}\right\}
\end{aligned}
$$

As before, we use $E^{*}$ to mean $E^{\text {dist }}$ resp. $E^{\text {prod }}$. 
Then by Chebyshev's inequality $\left|E \backslash E^{*}\right| \leq 2 \frac{|E|}{\lambda}$ for $q$ sufficiently large, since

$$
\begin{array}{r}
\left|E \backslash E^{d i s t}\right| \leq \frac{q}{\lambda|E|} \sum_{x} E * S_{t}(x) \leq 2 \frac{|E|}{\lambda}, \\
\left|E \backslash E^{p r o d}\right| \leq \frac{q}{\lambda|E|} \sum_{x, y} E(x) E(y) D_{t}(x, y) \leq 2 \frac{|E|}{\lambda}
\end{array}
$$

Note that any degenerate cycle is a connected graph on at most $k-1$ vertices, so it has a spanning tree which has the same number of vertices.

Definition 4.3. For a tree $T$ with vertex set $V$ and $|V| \leq n-1$, let $n_{T}^{*}$ be the number of edge-preserving maps of $T$ into $\mathcal{G}_{t}^{*}\left(E^{*}\right)$, and let $G_{T}$ be the number of graphs on $V$ with at most $n-1$ vertices for which $T$ is a spanning tree.

Then the number of degenerate $n$-cycles in $\mathcal{G}_{t}^{*}\left(E^{*}\right)$ is bounded by $\sum_{T} n_{T}^{*} G_{T}$, where the sum is taken over trees with at most $n-1$ vertices.

LEMMA 4.4. If $T$ has $r+1$ vertices and hence $r$ edges, then

$$
\left|n_{T}^{*}-\frac{|E|^{r+1}}{q^{r}}\right| \leq 4 r\left(\lambda^{-1}+\lambda^{\frac{r-1}{2}} \frac{q^{\frac{d+1}{2}}}{|E|}\right)
$$

Proof of Theorem 1.7. Setting $\lambda=q^{\frac{2 \varepsilon}{r+1}}$ in Lemma 4.4 yields Theorem 1.7 as a corollary.

Proof of Lemma 4.4. We proceed by induction on $r$, noting for the case $r=1$ that

$$
\left|n_{T}^{*}-\frac{\left|E^{*}\right|^{2}}{q}\right| \leq 2 q^{\frac{d-1}{2}}\left|E^{*}\right|
$$

Moreover $|E|-\left|E^{*}\right| \leq 2 \frac{|E|}{\lambda}$, so

$$
\begin{aligned}
\left|n_{T}^{*}-\frac{|E|^{2}}{q}\right| & \leq\left|n_{T}^{*}-\frac{\left|E^{*}\right|^{2}}{q}\right|+\left.\frac{1}{q}|| E\right|^{2}-\left|E^{*}\right|^{2} \mid \\
& \leq 2 q^{\frac{d-1}{2}}|E|+\frac{4|E|^{2}}{q \lambda}
\end{aligned}
$$

Fix a degree 1 vertex $v$ of $T$ with unique neighbor $w$, and let $T^{\prime}$ be the tree obtained from $T$ by deleting $v$, and the edge from $v$ to $w$. We will assume the result holds for $T^{\prime}$, and deduce that it holds for $T$. For $x \in E^{*}$, let $T^{\prime}(x)$ be the number of edge-preserving maps $\sigma: T^{\prime} \rightarrow \mathcal{G}_{t}^{*}\left(E^{*}\right)$ with $\sigma(w)=x$. Similarly to how we have defined $E^{*}$ and $G_{t}^{*}$, let $D_{t}^{*}(x, y)=D_{t}(x, y)$ in the dot-product case, and $D_{t}^{*}(x, y)=S_{t}(x-y)$ in the distance case. Then we have

$$
\begin{aligned}
n_{T}^{*} & =\sum_{x, y \in \mathbb{F}_{q}^{d}} E^{*}(y) D_{t}^{*}(x, y) T^{\prime}(x)=q^{-1}|| T^{\prime} \|_{1}\left|E^{*}\right|+R, \text { where } \\
R & \leq 2 q^{\frac{d-1}{2}}\left\|T^{\prime}\right\|_{2}\left|E^{*}\right|^{1 / 2}
\end{aligned}
$$

By our inductive assumption,

$$
\left|\left\|T^{\prime}\right\|_{1}-\frac{|E|^{r}}{q^{r-1}}\right| \leq(4 r-4) \frac{|E|^{r}}{q^{r-1}}\left(\lambda^{-1}+\lambda^{\frac{r-2}{2}} \frac{q^{\frac{d+1}{2}}}{|E|}\right)
$$


and in particular for $q$ sufficiently large we have $\left\|T^{\prime}\right\|_{1} \leq 2 \frac{|E|^{r}}{q^{r-1}}$. Thus,

$$
\begin{aligned}
\left|q^{-1}\right|\left|T^{\prime}\right|_{1}\left|E^{*}\right|-\frac{|E|^{r+1}}{q^{r}} \mid & \leq q^{-1}|| T^{\prime} \|_{1}\left(|E|-\left|E^{*}\right|\right)+\left|q^{-1}\right|\left|T^{\prime}\right|_{1}|E|-\frac{|E|^{r+1}}{q^{r}} \mid \\
& \leq \frac{|E|^{r+1}}{q^{r}}\left((4 r-2) \lambda^{-1}+(4 r-4) \lambda^{\frac{r-2}{2}} \frac{q^{\frac{d+1}{2}}}{|E|}\right)
\end{aligned}
$$

So to prove the lemma, it only remains to bound $R$, i.e. to bound $\left\|T^{\prime}\right\|_{2}$. Let $D$ be the maximum degree of a vertex in $\mathcal{G}_{t}^{*}\left(E^{*}\right)$, noting that by the definition of $E^{*}$,

$$
D=\max _{x \in E^{*}} \sum_{y} E^{*}(y) D_{t}^{*}(x, y) \leq \lambda \frac{|E|}{q}
$$

Also, trivially for any $x \in E^{*}, T^{\prime}(x) \leq D^{r-1}$. Therefore,

$$
\left\|T^{\prime}\right\|_{2}^{2} \leq \max _{x} T^{\prime}(x) \cdot\left\|T^{\prime}(x)\right\|_{1} \leq 2\left(\frac{\lambda|E|}{q}\right)^{r-1} \frac{|E|^{r}}{q^{r-1}}=2 \lambda^{r-1} \frac{|E|^{2 r-1}}{q^{2 r-2}}
$$

and thus

$$
R \leq 4 \lambda^{\frac{r-1}{2}} q^{\frac{d+1}{2}} \frac{|E|^{r}}{q^{r}}
$$

We conclude that

$$
\left|n_{T}^{*}-\frac{|E|^{r+1}}{q^{r}}\right| \leq 4 r \frac{|E|^{r+1}}{q^{r}}\left(\lambda^{-1}+\lambda^{\frac{r-1}{2}} \frac{q^{\frac{d+1}{2}}}{|E|}\right)
$$

completing the proof of the lemma.

COROLlary 4.5. With the notation from Theorem 1.7, if $|E| \geq q^{\frac{d+1}{2}+\varepsilon}$ then for sufficiently large $q$,

$$
n_{T}^{*} \leq 2 \frac{|E|^{r+1}}{q^{r}}
$$

Proof of Theorem 1.5. Let $E^{*}$ be defined as in Lemma 4.4 with $\lambda=q^{-\frac{2 \varepsilon}{k-1}},|E| \geq q^{\frac{d+1}{2}+\varepsilon}$, and

$$
\varepsilon= \begin{cases}1-\frac{k-2}{k-1}+\delta & : n=2 k \\ 1-\frac{2 k-3}{2 k-1}+\delta & : n=2 k+1\end{cases}
$$

Recall that the number of degenerate $n$-cycles in $\mathcal{G}_{t}^{*}\left(E^{*}\right)$ is bounded by $\sum_{T} n_{T}^{*} G_{T}$, where $G_{T}$ is the number of graphs on the same vertex set as $T$ for which $T$ is a spanning tree, and the sum is taken over trees with at most $n-1$ vertices. Now, $T$ has $r$ edges while the $K_{r+1}$ has $\left(\begin{array}{c}r+1 \\ 2\end{array}\right)$ edges. Since any subset of the edges in $K_{r+1}$ which are not in $T$ may be added to $T$ to form a graph whose spanning tree is $T$, we find that

$$
G_{T}=2^{\left(\begin{array}{c}
r+1 \\
2
\end{array}\right)-r}
$$

Furthermore, it was shown by Cayley in 1889 that the number of trees on $r+1$ vertices is $(r+1)^{r-1}$. Therefore, where $\mathcal{D}_{k}^{*}\left(E^{*}\right)$ is the number of degenerate cycles of length $n$ in $\mathcal{G}_{t}^{*}\left(E^{*}\right)$ and $\mathcal{N}_{k}^{*}\left(E^{*}\right)$ is 
the number of such non-degenerate cycles,

$$
\begin{aligned}
\mathcal{D}_{n}^{*}\left(E^{*}\right) & \leq \sum_{T} n_{T}^{*} G_{T}=\sum_{r=1}^{n-2} \sum_{|V|=r+1} n_{T}^{*} G_{T} \leq c_{n} \sum_{r=1}^{n-2} \frac{|E|^{r+1}}{q^{r}} \\
& =c_{n} \frac{|E|^{2}}{q} \cdot \frac{\frac{|E|^{n-2}}{q^{n-2}}-1}{\frac{|E|}{q}-1} \leq c_{n} \frac{|E|^{n-1}}{q^{n-2}},
\end{aligned}
$$

where $c_{n}=(n-1)^{n-3} \cdot 2^{\left(\begin{array}{c}n-1 \\ 2\end{array}\right)-n+3}$. Therfore,

$$
\begin{aligned}
\left|\mathcal{N}_{n}^{*}\left(E^{*}\right)-\frac{|E|^{n}}{q^{n}}\right| & \leq\left|C_{n}^{*}\left(E^{*}\right)-\frac{\left|E^{*}\right|^{n}}{q^{n}}\right|+q^{-n}\left(|E|^{n}-\left|E^{*}\right|^{n}\right)+\mathcal{D}_{n}^{*}\left(E^{*}\right) \\
& \leq K_{n} \frac{|E|^{n}}{q^{n}} q^{-\left(\frac{n}{2}-1\right) \delta}+n q^{-k}\left(|E|-\left|E^{*}\right|\right)|E|^{n-1}+c_{n} \frac{|E|^{n-1}}{q^{n-2}} \\
& \leq \frac{|E|^{n}}{q^{n}}\left(K_{n} q^{-\left(\frac{n}{2}-1\right) \delta}+2 n q^{-\frac{2}{n-1}}+c_{n} q^{-\frac{d-3}{2}-\varepsilon}\right)
\end{aligned}
$$

Now, since

$\frac{|E|^{n}}{q^{n}}\left(1-\left(K_{n} q^{-\left(\frac{n}{2}-1\right) \delta}+2 n q^{-\frac{2}{n-1}}+c_{n} q^{-\frac{d-3}{2}-\varepsilon}\right)\right) \leq \mathcal{N}_{n}^{*}\left(E^{*}\right) \leq \mathcal{N}_{n}^{*}(E) \leq C_{n}^{*}(E) \leq \frac{|E|^{n}}{q^{n}}\left(1+K_{n} q^{-\left(\frac{n}{2}-1\right) \delta}\right)$

We obtain the desired bounds for $N_{n}^{*}(E)$, and establish the existence of non-degenerate $n$-cycles when $d \geq 3$. 


\section{References}

[1] M. Bennett, J. Chapman, D. Covert, D. Hart, A. Iosevich, J. Pakianathan, Long Paths in the Distance Graph Over Large Subsets of Vector Spaces Over Finite Fields, J. Korean Math. Soc., 53, (2016), no. 1, 115-121.

[2] M. Bennett, D. Hart, A. Iosevich, J. Pakianathan, M. Rudnev, Group actions and geometric combinatorics in $\mathbb{F}_{q}^{d}$, Forum Math, 29, (2017), no. 1, 91-110.

[3] M. Bennett, A. Iosevich and J. Pakianathan, Three-point configurations determined by subsets of $\mathbb{F}_{q}^{2}$ via the Elekes-Sharir paradigm, Combinatorica, 34, (2014), no. 6, 689-706.

[4] J. Bourgain, N. Katz, and T. Tao A sum-product estimate in finite fields, and applications, Geom. Funct. Anal. 14 (2004), 27-57.

[5] P. Brass, W. Moser and J Pach, Research Problems in Discrete Geometry, Springer (2000).

[6] J. Chapman, M. B. Erdoğan, D. Hart, A. Iosevich and D. Koh, Pinned distance sets, $k$-simplices, Wolff's exponent in finite fields and sum-product estimates, Mathematische Zeitschrift, Math. Z. 271 (2012), no. 1-2, 63-93.

[7] D. Covert, D. Hart, A. Iosevich, D. Koh, M. Rudnev, Generalized Incidence Theorems, Homogeneous Forms and Sum-Product Estimates in Finite Fields, European J. Combin., 31, (2010), no. 1, 306-319.

[8] M. B. Erdoğan A bilinear Fourier extension theorem and applications to the distance set problem, IMRN (2006).

[9] A. Greenleaf, A. Iosevich and K. Taylor, Configuration sets with nonempty interior, (2019), arXiv:1907.12513.

[10] A. Greenleaf, A. Iosevich and K. Taylor, On k-point configuration sets with nonempty interior, arXiv:2005.10796

[11] L. Guth and N. Katz, On the Erdös distinct distance problem in the plane, Ann. of Math. (2), 181, (2015), no. 1, 155-190.

[12] D. Hart and A. Iosevich, Sums and products in finite fields: an integral geometric view- point, Contemporary Mathematics: Radon transforms, geometry, and wavelets, 464, (2008).

[13] D. Hart, A. Iosevich, D. Koh and M. Rudnev, Averages over hyperplanes, sum-product theory in finite fields, and the Erdös-Falconer distance conjecture, Transactions of the AMS, 363 (2011) 3255-3275.

[14] A. Iosevich and H. Parshall, Embedding distance graphs in finite field vector spaces, J. Korean Math. Soc. 56 (2019), no. 6, 1515-1528.

[15] A. Iosevich and M. Rudnev Erdös distance problem in vector spaces over finite fields, Trans. Amer. Math. Soc. 359 (2007), no. 12, 6127-6142.

[16] A. Iosevich, K. Taylor and I. Uriarte-Tuero, Pinned geometric configurations in Euclidean space and Riemannian manifolds, (2018), arXiv:1610.00349.

[17] P. Mattila Spherical averages of Fourier transforms of measures with finite energy: dimensions of intersections and distance sets Mathematika, 34 (1987), 207-228.

[18] P. Mattila, Geometry of sets and measures in Euclidean spaces, Cambridge University Press, volume 44, (1995).

[19] H. Minkowski, Grundlagen für eine Theorie quadratischen Formen mit ganzahligen Koeffizienten, Gesammelte Abhandlungen, 3-145, (1911).

[20] D. Pham, T. Pham, Thang, and L. Vinh, An improvement on the number of simplices in $\mathbb{F}_{q}^{d}$, Discrete Appl. Math. 221 (2017), 95-105.

[21] D. Phong and E. Stein, Radon transforms and torsion, Internat. Math. Res. Notices (1991), no. 4, 49-60.

[22] Y. Shteinikov, Long paths in the distance graphs in vector spaces over finite fields, Chebyshevskii Sbornik, volume 19, Issue 3, (2018,) pages 311-317.

[23] C. Sogge, Fourier integrals in classical analysis, Cambridge University Press, (1993).

[24] J. Solymosi and V. Vu, Distinct distances in high dimensional homogeneous sets, Towards a theory of geometric graphs, 259-268, Contemp. Math. 342, Amer. Math. Soc., Providence, 2004.

[25] Le Anh Vinh, On a Furstenberg-Katznelson-Weiss type theorem over finite fields, Ann. Comb. 15 (2011), no. $3,541-547$

Department of Mathematics, University of Rochester, Rochester, NY

Email address: iosevich@math.rochester.edu

Email address: gjardine@ur.rochester.edu

Email address: bmcdon11@ur.rochester.edu 